\title{
LQG Control of Networked Control Systems with Limited Information
}

\author{
Qing-Quan Liu ${ }^{1,2}$ and Fang Jin ${ }^{1}$ \\ ${ }^{1}$ College of Equipment Engineering, Shenyang Ligong University, Shenyang 110168, China \\ ${ }^{2}$ Shenyang Institute of Automation, Chinese Academy of Sciences, Shenyang 110016, China \\ Correspondence should be addressed to Qing-Quan Liu; lqqneu@163.com
}

Received 1 August 2014; Accepted 26 October 2014; Published 13 November 2014

Academic Editor: Hak-Keung Lam

Copyright (C) 2014 Q.-Q. Liu and F. Jin. This is an open access article distributed under the Creative Commons Attribution License, which permits unrestricted use, distribution, and reproduction in any medium, provided the original work is properly cited.

This paper addresses linear quadratic Gaussian (LQG) control problems for multi-input multioutput (MIMO), linear time-invariant (LTI) systems, where the sensors and controllers are geographically separated and connected via a digital communication channel with limited data rates. An observer-based, quantized state feedback control scheme is employed in order to achieve the minimum data rate for mean square stabilization of the unstable plant. An explicit expression is presented to state the tradeoff between the LQ cost and the data rate. Sufficient conditions on the data rate for mean square stabilization are derived. An illustrative example is given to demonstrate the effectiveness of the proposed scheme.

\section{Introduction}

In recent years, networked control systems have attracted unprecedented attention of the control community in view of their wide applications in many fields such as vehicle control systems, large-scale printers, and aerospace applications. In such systems, the sensors, actuators, and controllers are geographically separated and connected via digital wireless channels such as the Internet or bus lines. Using networked control offers many advantages, such as increased system flexibility, ease of installation and maintenance, and decreased wiring and cost. However, the presence of digital communication channels brings up many challenges. Communication constraints often make traditional control approaches inefficient $[1,2]$.

In particular, the problem of control under data-rate limitations has been the focus of many researches. A highwater mark in the study of quantized feedback using data rate limited feedback channels is known as the data rate theorem that states that the larger the magnitude of the unstable poles, the larger the required data rate through the feedback loop $[3,4]$.

There has been a lot of research on quantized feedback control. It was shown in [5] that there exists a dynamic adjustment of the quantizer sensitivity and a quantized state feedback that stabilizes linear time-invariant systems without disturbances. The problem of LQG control under communication constraints was addressed in [6]. There they looked at stable systems and noiseless digital channels and introduced the new coding scheme. Tatikonda at al. [7] examined the role communication has on the classical LQG control problem and designed the encoder, decoder, and controller to satisfy some given control objective. Imer and Başar [8] considered optimal LQG control of a scalar system with limited control actions. Schenato et al. [9] considered the problem of LQG control over a packet-dropping network. Furthermore, Bommannavar and Başar [10] addressed optimal LQG control of higher-order systems with limited control actions. It was shown that the optimal control is a threshold policy. Furthermore, in [11], the LQG control problem for stochastic linear control systems was addressed. In particular, the sequential rate distortion framework was presented and the inherent tradeoffs between control and communication costs was shown in [7]. The optimal LQ cost is decomposed into two terms: a full knowledge cost and a sequential rate distortion cost. However, the second term still depends on the steady state estimation error covariance. Differently from the existing ones, it is shown in our results that the steady state estimation error depends on the disturbances, and a full knowledge LQ cost is presented. Furthermore, Georges et al. 


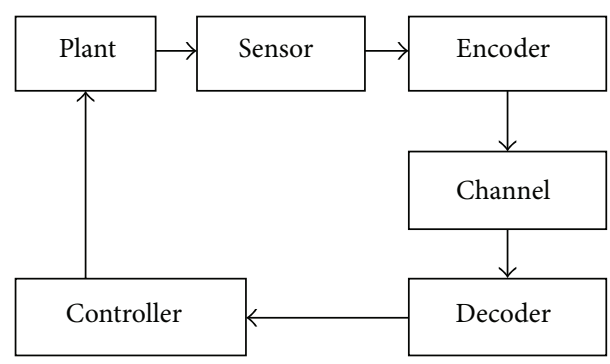

FIGURE 1: Networked control systems.

[12] gave the design of a decentralized optimal batch LQ state observer for state estimation of large-scale interconnected systems, and Wang and Han [13] was concerned with modelling and controller design for a discrete-time networked control system with limited channels and data drift.

This paper considers a class of networked control problems which arises in the coordinated motion control of autonomous and semiautonomous mobile agents, for example, unmanned air vehicles (UAVs), unmanned ground vehicles (UGVs), and unmanned underwater vehicles (UUVs). The controller often lies in such unmanned vehicles, but some sensors (such as automatic radar measuring equipment) are on the ground and are connected with the controllers via noisy, bandwidth-limited wireless communication channels. However, as the data rate of the channel is reduced to the critical value, the plant states must always become unbounded. Then, in engineering systems, it is of importance to present a lower bound of the data rate above which there exists a quantization, coding and control scheme to guarantee some given control performances.

The aforementioned results considered the fully observed systems. This paper considers a partially observed, linear time-invariant system and employs an observer-based quantized state feedback control scheme in order to achieve the minimum data rate for mean square stabilization of the unstable plant. Furthermore, LQG control problems are further addressed under data-rate limitations. It is shown that there exists the tradeoff between the LQ cost and the data rate. Then, an explicit expression on the tradeoff is presented in our results.

The rest of the paper is organized as follows. In Section 2, the problem formulation is presented. Section 3 deals with LQG control problems under data-rate limitations. The results of numerical simulation are presented in Section 4. Conclusions are stated in Section 5.

\section{Problem Formulation}

Consider the following discrete linear time-invariant system in Figure 1. The system dynamics is given by

$$
\begin{gathered}
X(k+1)=A X(k)+B U(k)+F W(k), \\
Y(k)=C X(k),
\end{gathered}
$$

where $X(k) \in R^{n}$ denotes the plant state, $U(k) \in R^{m}$ denotes the control input, $Y(k) \in R^{p}$ denotes the observation output, and $W(k) \in R^{q}$ denotes the disturbance, respectively. The initial position $X(0)$ and $W(k)$ are mutually independent, Gaussian random variables with zero mean, satisfying

$$
\begin{gathered}
E\|X(0)\|^{2}<\phi_{0}<\infty, \\
E\|W(k)\|^{2}<\phi_{W}<\infty .
\end{gathered}
$$

$A, B, C$, and $F$ are known constant matrices with appropriate dimensions. Without loss of generality, assume that the pair $(A, B)$ is a controllable pair, and the pair $(A, C)$ is an observable pair.

In the MIMO case, it seems logical to try to implement a quantized output feedback control law of the form

$$
U(k)=K q(Y(k)),
$$

where $q(\cdot)$ denotes a quantizer and $K$ denotes the feedback gain. However, it is difficult to find a bit-allocation algorithm which can regulate the transmission of information about each $y_{i}(k)(i=1,2, \ldots, p)$ since $y_{i}(k)$ and $y_{j}(k)(i \neq j)$ often are mutually correlated. Namely, it leads to the larger data rate for stabilization. Thus, this paper implements an observerbased quantized state feedback control law of the form

$$
\begin{gathered}
X_{o}(k+1)=A X_{o}(k)+B U(k)+L\left(Y(k)-C X_{o}(k)\right), \\
U(k)=K \widehat{X}_{o}(k),
\end{gathered}
$$

where $L$ denotes the observer gain and $\widehat{X}_{o}(k)$ denotes the estimate of $X_{o}(k)$ at the decoder. Here, define

$$
D(k):=X(k)-X_{o}(k) .
$$

Then, it follows from (4) that

$$
X_{o}(k+1)=A X_{o}(k)+B U(k)+L C D(k) .
$$

By summing (1) and (6), obtain that

$$
D(k+1)=(A-L C) D(k)+F W(k) .
$$

Here, assume that there exists an observer gain $L$ such that all eigenvalues of $A-L C$ lie inside the unit circle. Then, it holds that

$$
\limsup _{T \rightarrow \infty} \frac{1}{T} \sum_{k=0}^{T-1} E\left[D^{\prime}(k) Q D(k)\right]<\frac{\left\|Q^{1 / 2} F\right\|^{2}}{1-\|A-L C\|^{2}} \phi_{W} .
$$

In the literature, many works were concerned with networked control over dropout channels, or the channels with time delays, and presented many important results. Differently from the existing results, this paper considers the case where the sensors and the controller are connected via errorless digital communication channels without time delays and focuses on the tradeoff between the LQ cost and the data rate of the channel. Furthermore, assume that the channel is memoryless. Then, the encoder and the decoder have access to the control actions. Considering this case avoids extraneous complexity. It makes our conclusions most transparent. 
This paper considers the MIMO system (1) under datarate limitations, and presents a quantization, coding, and control scheme to stabilize the system (1) in the mean square sense

$$
\limsup _{T \rightarrow \infty} \frac{1}{T} \sum_{k=0}^{T-1} E\|X(k)\|^{2}<\infty
$$

Furthermore, LQG control problems are also further discussed under data-rate limitations, and the role that communication has on the classical LQG control problem is explicitly examined. The main task here is to present an explicit expression on the tradeoff between the LQ cost and the data rate.

\section{LQG Control for MIMO Systems under Data-Rate Limitations}

This section considers the partially-observed, unstable linear time-invariant plant, discusses the LQG control problem under data-rate limitations, derives the sufficient condition on the data rate for stabilization, and presents the relationship between the control performance and the data rate.

In order to achieve the minimum data rate for stabilization, the existing bit-allocation scheme needs to find a real transformation matrix which can diagonalize the system matrix, such that it can regulate the transmission of information on the basis of the eigenvalues. In many applications, however, there exists no transformation matrix that can diagonalize any system matrix. Furthermore, putting the system matrix into Jordan canonical form often requires a complex transformation matrix. To solve the problem, a bit-allocation scheme on the basis of the singular values of system matrix and an adaptive differential quantizationcoding scheme are employed in this paper.

Notice that there must exist a real orthogonal matrix $H \in$ $R^{n \times n}$ that diagonalizes

$$
A^{\prime} A=H^{\prime} \Lambda^{2} H
$$

where we define $\Lambda:=\operatorname{diag}\left[\sigma_{1}, \ldots, \sigma_{n}\right]$. Clearly, $\sigma_{i}$ is the $i$ th singular value of $A(i=1,2, \ldots, n)$. Here, define the prediction value of $X_{o}(k)$ by

$$
\grave{X}_{o}(k):=(A+B K) \widehat{X}_{o}(k-1) .
$$

Furthermore, define

$$
\begin{gathered}
\bar{X}_{o}(k):=H X_{o}(k), \\
\widetilde{X}_{o}(k):=H \widehat{X}_{o}(k), \\
\vec{X}_{o}(k):=H \grave{X}_{o}(k), \\
Z(k):=\bar{X}_{o}(k)-\vec{X}_{o}(k) .
\end{gathered}
$$

Since the encoder and the decoder have access to the previous control actions, update their estimator, and obtain the same prediction value, only $Z(k)$ needs to be quantized, encoded, and transmitted to the decoder. Let $\widehat{Z}(k)$ and $V(k)$ denote the quantization value and quantization error of $Z(k)$, respectively. Then, it follows that

$$
Z(k)=\widehat{Z}(k)+V(k) .
$$

Thus, the estimate of $X_{o}(k)$ is given by

$$
\widehat{X}_{o}(k)=H^{\prime}\left(\vec{X}_{o}(k)+\widehat{Z}(k)\right) \text {. }
$$

Similar to that in [5], the quantization scheme is presented. Let $Z(t):=\left[\begin{array}{lllll}z_{1}(t) & z_{2}(t) & \cdots & z_{n}(t)\end{array}\right]^{\prime}$. Given a positive integer $M_{i}$ and a nonnegative real number $\Delta_{i}(t)(i=$ $1, \ldots, n)$, define the quantizer $q: R \rightarrow Z$ with sensitivity $\Delta_{i}(t)$ and saturation value $M_{i}$ by the formula

$$
\begin{aligned}
& q\left(z_{i}(t)\right) \\
& = \begin{cases}M^{+}, & \text {if } z_{i}(t)>\left(M_{i}+\frac{1}{2}\right) \Delta_{i}(t) \\
M^{-}, & \text {if } z_{i}(t) \leq-\left(M_{i}+\frac{1}{2}\right) \Delta_{i}(t) \\
\left\lfloor\frac{z_{i}(t)}{\Delta_{i}(t)}+\frac{1}{2}\right\rfloor, & \text { if }-\left(M_{i}+\frac{1}{2}\right) \Delta_{i}(t)<z_{i}(t),\end{cases}
\end{aligned}
$$

where we define $\lfloor z\rfloor:=\max \{k \in Z:=k<z, z \in R\}$. The indexes $M^{+}$and $M^{-}$will be employed if the quantizer saturates. The scheme to be used here is based on the hypothesis that it is possible to change the sensitivity (but not the saturation value) of the quantizer on the basis of available quantized measurements. The quantizer may counteract disturbances by switching repeatedly between "zooming out" and "zooming in."

First, a lemma from [14] is presented.

Lemma 1. Let $z \in R$ denote a Gaussian source and $\widehat{z}$ denote an estimate of $z$. Define $R(D)$ as the data rate distortion function between $\widehat{z}$ and $z$. The distortion constraint is defined as $D \in R^{+}$. Let $h$ denote the sampling period. Given $D \geq E(z-\widehat{z})^{2}$, there must exist a quantization and coding scheme if the information rate $R$ of the channel satisfies

$$
R>\frac{1}{h} R(D) \geq \frac{1}{2} \log _{2} \frac{\sigma^{2}(z)}{D}(\text { bits/sample }),
$$

where one defines $\sigma^{2}(z):=E(z-E z)^{2}$.

Proof. The proof is given by [14].

In networked control systems with large communication bandwidth, communication and control are often viewed as independent functions in order to simplify the analysis and design of the overall system. However, in many applications, data-rate limitations can introduce large quantization errors and affect control performances significantly. Thus, this paper is concerned with the relationship between the control performance and the data rate.

Here, the LQ cost is quantified by

$$
J_{1}=\limsup _{T \rightarrow \infty} \frac{1}{T} \sum_{k=0}^{T-1} E\left[X^{\prime}(k) Q X(k)\right],
$$


where $Q \in R^{n \times n}$ is symmetric positive definite. Here, this paper is concerned with how small the plant state can be made as $k \rightarrow \infty$. Then, the following result holds.

Theorem 2. Consider the system (1). Assume that all eigenvalues of $A+B K$ and $A-L C$ lie inside the unit circle. Then, the system (1) is stabilizable in the mean square sense (9) if the data rate of the channel satisfies the following condition:

$$
R>R_{\min }=\sum_{i \in \Xi} \log _{2}\left|\sigma_{i}\right|(\text { bits } / \text { sample })
$$

with $\Xi:=\left\{i \in\{1,2, \ldots, n\}:\left|\sigma_{i}\right|>1\right\}$. If one further assumes that the magnitudes of all the singular values of system matrix $A$ are larger than 1, the system (1) is stabilizable in the mean square sense (9) if the data rate of the channel satisfies the following condition:

$$
R>R_{\min }=\log _{2}|A| \text { (bits/sample). }
$$

Furthermore, the LQ cost $J_{1}$ is obtained by

$$
\begin{aligned}
J_{1}= & \limsup _{T \rightarrow \infty} \frac{1}{T} \sum_{k=0}^{T-1} E\left[X^{\prime}(k) Q X(k)\right] \\
< & \frac{1}{1-\|A-L C\|^{2}} \\
& \times\left[\frac{1}{\left(1-\sqrt[n]{|A|^{2} / 4^{\bar{R}}}\right)\left(1-\|A+B K\|^{2}\right)}\right. \\
& \times\left(\sqrt[n]{\frac{|A|^{2}}{4^{\bar{R}}}}\left\|Q^{1 / 2}(A+B K) A^{-1} L C F\right\|^{2}+\left\|Q^{1 / 2} L C F\right\|^{2}\right. \\
& +\frac{\left.2\left\|F^{\prime} \sqrt{\frac{|A|^{2}}{4^{\bar{R}}}}\right\| F^{\prime}(L C)^{\prime}(A+B K)^{\prime} Q A^{-1} L C F \|\right)}{\left(1-\|A+B K\|^{2}\right)\left(1-\left\|(A+B K)^{\prime}(A-L C)\right\|\right)} \\
& \left.+\left\|Q^{1 / 2} F\right\|^{2}+\frac{2\left\|F^{\prime}(A-L C)^{\prime} Q L C F\right\|}{1-\left\|(A+B K)^{\prime}(A-L C)\right\|}\right] \phi_{W} .
\end{aligned}
$$

Proof. Notice that

$$
X(k)=X_{o}(k)+D(k) .
$$

Then, it holds that

$$
\begin{aligned}
E\left[X^{\prime}(k) Q X(k)\right]= & E\left[X_{o}^{\prime}(k) Q X_{o}(k)\right] \\
& +E\left[D^{\prime}(k) Q D(k)\right] \\
& +2 E\left[X_{o}^{\prime}(k) Q D(k)\right] .
\end{aligned}
$$

Furthermore, notice that

$$
X_{o}(k)=\grave{X}_{o}(k)+H^{\prime} Z(k) .
$$

Then, it follows that

$$
\begin{aligned}
& E\left[X_{o}^{\prime}(k) Q X_{o}(k)\right] \\
&=E\left[\left(\grave{X}(k)+H^{\prime} Z(k)\right)^{\prime} Q\left(\grave{X}(k)+H^{\prime} Z(k)\right)\right] \\
&=E\left[\grave{X}_{o}^{\prime}(k) Q \grave{X}_{o}(k)\right]+E\left[Z^{\prime}(k) H Q H^{\prime} Z(k)\right] \\
&+2 E\left[\vec{X}_{o}(k) H Q H^{\prime} Z(k)\right] .
\end{aligned}
$$

Thus, it follows that

$$
\begin{aligned}
& E\left[X^{\prime}(k) Q X(k)\right] \\
&= E\left[\grave{X}_{o}^{\prime}(k) Q \grave{X}_{o}(k)\right]+E\left[Z^{\prime}(k) H Q H^{\prime} Z(k)\right] \\
&+2 E\left[\vec{X}_{o}(k) H Q H^{\prime} Z(k)\right]+E\left[D^{\prime}(k) Q D(k)\right] \\
&+2 E\left[X_{o}^{\prime}(k) Q D(k)\right] .
\end{aligned}
$$

Namely, it holds that

$$
\begin{aligned}
& \limsup _{T \rightarrow \infty} \frac{1}{T} \sum_{k=0}^{T-1} E\left[X^{\prime}(k) Q X(k)\right] \\
& =\limsup _{T \rightarrow \infty} \frac{1}{T} \sum_{k=0}^{T-1} E\left[\grave{X}_{o}^{\prime}(k) Q \grave{X}_{o}(k)\right] \\
& +\limsup _{T \rightarrow \infty} \frac{1}{T} \sum_{k=0}^{T-1} E\left[Z^{\prime}(k) H Q H^{\prime} Z(k)\right] \\
& +2 \limsup _{T \rightarrow \infty} \frac{1}{T} \sum_{k=0}^{T-1} E\left[\vec{X}_{o}(k) H Q H^{\prime} Z(k)\right] \\
& +\limsup _{T \rightarrow \infty} \frac{1}{T} \sum_{k=0}^{T-1} E\left[D^{\prime}(k) Q D(k)\right] \\
& +2 \limsup _{T \rightarrow \infty} \frac{1}{T} \sum_{k=0}^{T-1} E\left[X_{o}^{\prime}(k) Q D(k)\right] .
\end{aligned}
$$

Clearly, it needs to compute all the terms in the equation above.

It follows from (4) and (6) that

$$
\begin{aligned}
X_{o}(k & +1) \\
& =A X_{o}(k)+B K \widehat{X}_{o}(k)+L C D(k) \\
& =A\left(X_{o}(k)-\widehat{X}_{o}(k)\right)+(A+B K) \widehat{X}_{o}(k)+L C D(k) \\
& =A H^{\prime} V(k)+\grave{X}_{o}(k+1)+L C D(k) .
\end{aligned}
$$


Namely, it holds that

$$
Z(k+1)=H A H^{\prime} V(k)+H L C D(k) .
$$

Notice that $V(k)$ and $D(k)$ are mutually independent random variables. Let $X$ denote a vector. Then, define

$$
\Sigma_{X}:=E\left[X X^{\prime}\right]
$$

Then, it follows that

$$
\begin{aligned}
& E\left[Z^{\prime}(k+1) H Q H^{\prime} Z(k+1)\right] \\
& \quad=\operatorname{tr}\left(H Q H^{\prime} \Lambda^{2} \Sigma_{V(k)}\right)+E\left\|Q^{1 / 2} L C D(k)\right\|^{2} .
\end{aligned}
$$

Since $Z(k)$ is unknown for the decoder, it will be quantized, encoded, and transmitted via a digital communication channel with limited data rates. If there exists a quantization, coding scheme such that the following condition holds,

$$
E\left[z_{i}^{2}(k)\right]>\sigma_{i}^{2} E\left[v_{i}^{2}(k)\right] \quad(i=1,2, \ldots, n),
$$

which is equivalent to

$$
\varepsilon^{2} E\left[z_{i}^{2}(k)\right]=\sigma_{i}^{2} E\left[v_{i}^{2}(k)\right] \quad(i=1,2, \ldots, n)
$$

with $\varepsilon \in(0,1)$, it holds that

$$
\varepsilon^{2} E\left[Z^{\prime}(k) H Q H^{\prime} Z(k)\right]=\operatorname{tr}\left(H Q H^{\prime} \Lambda^{2} \Sigma_{V(k)}\right) .
$$

Substitute the equality above into (30), and obtain

$$
\begin{aligned}
& E\left[Z^{\prime}(k+1) H Q H^{\prime} Z(k+1)\right] \\
& \quad=\varepsilon^{2} E\left[Z^{\prime}(k) H Q H^{\prime} Z(k)\right]+E\left\|Q^{1 / 2} L C D(k)\right\|^{2} .
\end{aligned}
$$

It means that

$$
\begin{aligned}
\limsup _{T \rightarrow \infty} & \frac{1}{T} \sum_{k=0}^{T-1} E\left[Z^{\prime}(k) H Q H^{\prime} Z(k)\right] \\
& <\frac{1}{1-\varepsilon^{2}} \limsup _{T \rightarrow \infty} \frac{1}{T} \sum_{k=0}^{T-1} E\left\|Q^{1 / 2} L C D(k)\right\|^{2} \\
& <\frac{\left\|Q^{1 / 2} L C F\right\|^{2}}{\left(1-\varepsilon^{2}\right)\left(1-\|A-L C\|^{2}\right)} \phi_{W} .
\end{aligned}
$$

Clearly, the condition (31) must hold if the number of bits needed to the quantization value is large enough. Let $D_{i}(k)$ denote the expected distortion constraint corresponding to $z_{i}(k)$ at time $k$. Thus, set

$$
D_{i}(k)= \begin{cases}\frac{\varepsilon^{2}}{\sigma_{i}^{2}} E\left[z_{i}^{2}(k)\right], & \text { when }\left|\sigma_{i}\right| \geq 1 \\ 0, & \text { when }\left|\sigma_{i}\right|<1\end{cases}
$$

Then, it follows from Lemma 1 that the data rate of the channel is given by

$$
\begin{aligned}
R & =\frac{1}{2} \sum_{i \in \Xi} \log _{2} \frac{E\left[z_{i}^{2}(k)\right]}{D_{i}(k)} \\
& =\frac{1}{2} \sum_{i \in \Xi} \log _{2} \frac{E\left[z_{i}^{2}(k)\right]}{\left(\varepsilon^{2} / \sigma_{i}^{2}\right) E\left[z_{i}^{2}(k)\right]} \\
& =\sum_{i \in \Xi} \log _{2} \frac{\left|\sigma_{i}\right|}{\varepsilon} \text { (bits/sample), }
\end{aligned}
$$

where we define $\Xi:=\left\{i \in\{1,2, \ldots, n\}:\left|\sigma_{i}\right|>1\right\}$. A lower bound on the data rate which ensures the condition (31) holds is given by

$$
R_{\min }=\sum_{i \in \Xi} \log _{2}\left|\sigma_{i}\right| \text { (bits/sample) }
$$

If we assume that the magnitudes of all the singular values of system matrix $A$ are larger than 1, the data rate of the channel is given by

$$
R=\log _{2} \frac{|A|}{\varepsilon^{n}} \text { (bits/sample) }
$$

and the lower bound on the data rate is given by

$$
R_{\min }=\log _{2}|A| \text { (bits/sample). }
$$

It may be also obtained that

$$
\varepsilon=\sqrt[n]{\frac{|A|}{2^{\bar{R}}}} .
$$

Furthermore, it follows from (6) that

$$
\begin{aligned}
X_{o}(k+1) & =A X_{o}(k)+B K \widehat{X}_{o}(k)+L C D(k) \\
& =(A+B K) X_{o}(k)-B K H^{\prime} V(k)+L C D(k) .
\end{aligned}
$$

By summing the equality above and (7), it follows that

$$
\begin{aligned}
& E\left[X_{o}^{\prime}(k+1) Q D(k+1)\right] \\
& =E\left[X_{o}^{\prime}(k)(A+B K)^{\prime} Q(A-L C) D(k)\right] \\
& \quad+E\left[D^{\prime}(k)(A-L C)^{\prime} Q L C D(k)\right] .
\end{aligned}
$$


Thus, it follows that

$$
\begin{aligned}
\limsup _{T \rightarrow \infty} & \frac{1}{T} \sum_{k=0}^{T-1} E\left[X_{o}^{\prime}(k) Q D(k)\right] \\
& <\frac{\left\|F^{\prime}(A-L C)^{\prime} Q L C F\right\|}{\left(1-\left\|(A+B K)^{\prime}(A-L C)\right\|\right)\left(1-\|A-L C\|^{2}\right)} \phi_{W} .
\end{aligned}
$$

Using the same techniques in the proof above, it can be shown that

$$
\begin{aligned}
\limsup _{T \rightarrow \infty} & \frac{1}{T} \sum_{k=0}^{T-1} E\left[X_{o}^{\prime}(k)(A+B K)^{\prime} Q L C D(k)\right] \\
& <\frac{\left\|F^{\prime}(L C)^{\prime}(A-L C)^{\prime} Q(A+B K) L C F\right\|}{\left(1-\left\|(A+B K)^{\prime}(A-L C)\right\|\right)\left(1-\|A-L C\|^{2}\right)} \phi_{W} .
\end{aligned}
$$

It follows from (31) that

$$
\begin{array}{r}
\varepsilon^{2}\left[H(A+B K)^{\prime} Q A H^{\prime}\right]_{i, i} \sigma_{i}^{-2} E\left[z_{i}^{2}(k)\right] \\
=\left[H(A+B K)^{\prime} Q A H^{\prime}\right]_{i, i} E\left[v_{i}^{2}(k)\right],
\end{array}
$$

where $[\cdot]_{i, j}$ denotes an element of a matrix $(i, j=1,2, \ldots, n)$. This implies that

$$
\begin{aligned}
\varepsilon^{2} E\left[Z^{\prime}(k) H(A+B K)^{\prime} Q A^{-1} H^{\prime} Z(k)\right] \\
=E\left[V^{\prime}(k) H(A+B K)^{\prime} Q A H^{\prime} V(k)\right] .
\end{aligned}
$$

Furthermore, it follows from (28) that

$$
\begin{aligned}
& E\left[Z^{\prime}(k+1) H(A+B K)^{\prime} Q A^{-1} H^{\prime} Z(k+1)\right] \\
&= E\left[V^{\prime}(k) H(A+B K)^{\prime} Q A H^{\prime} V(k)\right] \\
&+E\left[D^{\prime}(k)(L C)^{\prime} H(A+B K)^{\prime} Q A^{-1} H^{\prime} L C D(k)\right] .
\end{aligned}
$$

Substitute (47) into the equality above, and obtain

$$
\begin{aligned}
& E\left[Z^{\prime}(k+1) H(A+B K)^{\prime} Q A^{-1} H^{\prime} Z(k+1)\right] \\
&= \varepsilon^{2} E\left[Z^{\prime}(k) H(A+B K)^{\prime} Q A^{-1} H^{\prime} Z(k)\right] \\
&+E\left[D^{\prime}(k)(L C)^{\prime} H(A+B K)^{\prime} Q A^{-1} H^{\prime} L C D(k)\right] .
\end{aligned}
$$

Then, get

$$
\begin{gathered}
\limsup _{T \rightarrow \infty} \frac{1}{T} \sum_{k=0}^{T-1} E\left[Z^{\prime}(k) H(A+B K)^{\prime} Q A^{-1} H^{\prime} Z(k)\right] \\
<\frac{\left\|F^{\prime}(L C)^{\prime}(A+B K)^{\prime} Q A^{-1} L C F\right\|}{\left(1-\varepsilon^{2}\right)\left(1-\|A-L C\|^{2}\right)} \phi_{W} .
\end{gathered}
$$

Thus, it holds that

$$
\begin{gathered}
\limsup _{T \rightarrow \infty} \frac{1}{T} \sum_{k=0}^{T-1} E\left[V^{\prime}(k) H(A+B K)^{\prime} Q A H^{\prime} V(k)\right] \\
<\frac{\varepsilon^{2}\left\|F^{\prime}(L C)^{\prime}(A+B K)^{\prime} Q A^{-1} L C F\right\|}{\left(1-\varepsilon^{2}\right)\left(1-\|A-L C\|^{2}\right)} \phi_{W} .
\end{gathered}
$$

Using the same techniques in the proof above, it can be shown that

$$
\begin{aligned}
\limsup _{T \rightarrow \infty} & \frac{1}{T} \sum_{k=0}^{T-1} E\left[V^{\prime}(k) H(A+B K)^{\prime} Q(A+B K) H^{\prime} V(k)\right] \\
< & \frac{\varepsilon^{2}\left\|Q^{1 / 2}(A+B K) A^{-1} L C F\right\|^{2}}{\left(1-\varepsilon^{2}\right)\left(1-\|A-L C\|^{2}\right)} \phi_{W} .
\end{aligned}
$$

Furthermore, notice that

$$
\begin{gathered}
\vec{X}_{o}(k)=H(A+B K)\left(X_{o}(k-1)-H^{\prime} V(k-1)\right), \\
Z(k)=H A H^{\prime} V(k-1)+H L C D(k-1) .
\end{gathered}
$$

Then, it follows that

$$
\begin{aligned}
& E\left[\vec{X}_{o}(k) H Q H^{\prime} Z(k)\right] \\
& =E\left[\left(X_{o}(k-1)-H^{\prime}\right)^{\prime}(A+B K)^{\prime} Q\right. \\
& \left.\quad \times\left(A H^{\prime} V(k-1)+L C D(k-1)\right)\right] \\
& =E\left[X_{o}^{\prime}(k-1)(A+B K)^{\prime} Q L C D(k-1)\right] \\
& \quad-E\left[V^{\prime}(k-1) H(A+B K)^{\prime} Q A H^{\prime} V(k-1)\right] .
\end{aligned}
$$

Substitute (45) and (51) into the previous equality, and obtain

$$
\begin{aligned}
\limsup _{T \rightarrow \infty} \frac{1}{T} \sum_{k=0}^{T-1} E\left[\vec{X}_{o}(k) H Q H^{\prime} Z(k)\right] \\
<\frac{\varepsilon^{2}\left\|F^{\prime}(L C)^{\prime}(A+B K)^{\prime} Q A^{-1} L C F\right\|}{\left(1-\varepsilon^{2}\right)\left(1-\|A-L C\|^{2}\right)} \phi_{W} \\
+\frac{\left\|F^{\prime}(L C)^{\prime}(A-L C)^{\prime} Q(A+B K) L C F\right\|}{\left(1-\left\|(A+B K)^{\prime}(A-L C)\right\|\right)\left(1-\|A-L C\|^{2}\right)} \phi_{W} .
\end{aligned}
$$


Furthermore, notice that

$$
\begin{aligned}
\grave{X}_{o}(k) & =(A+B K) \widehat{X}_{o}(k-1) \\
& =(A+B K)\left(X_{o}(k-1)-H^{\prime} V(k-1)\right) .
\end{aligned}
$$

Then, it holds that

$$
\begin{gathered}
\limsup _{T \rightarrow \infty} \frac{1}{T} \sum_{k=0}^{T-1} E\left[\grave{X}_{o}^{\prime}(k) Q \grave{X}_{o}(k)\right] \\
=\limsup _{T \rightarrow \infty} \frac{1}{T} \sum_{k=0}^{T-1} E\left[X_{o}^{\prime}(k-1)(A+B K)^{\prime}\right. \\
\left.\times Q(A+B K) X_{o}(k-1)\right] \\
+\limsup _{T \rightarrow \infty} \frac{1}{T} \sum_{k=0}^{T-1} E\left[V^{\prime}(k-1) H(A+B K)^{\prime}\right. \\
\left.\times Q(A+B K) H^{\prime} V(k-1)\right] \\
<\|A+B K\|^{2} \limsup _{T \rightarrow \infty} \frac{1}{T} \sum_{k=0}^{T-1} E\left[X_{o}^{\prime}(k) Q X_{o}(k)\right] \\
+\limsup _{T \rightarrow \infty} \frac{1}{T} \sum_{k=0}^{T-1} E\left[V^{\prime}(k) H(A+B K)^{\prime}\right. \\
\left.\times Q(A+B K) H^{\prime} V(k)\right] .
\end{gathered}
$$

Substitute (52) into the inequality above, and obtain that

$$
\begin{aligned}
& \limsup _{T \rightarrow \infty} \frac{1}{T} \sum_{k=0}^{T-1} E\left[\grave{X}_{o}^{\prime}(k) Q \grave{X}_{o}(k)\right] \\
&<\|A+B K\|^{2} \limsup _{T \rightarrow \infty} \frac{1}{T} \sum_{k=0}^{T-1} E\left[X_{o}^{\prime}(k) Q X_{o}(k)\right] \\
&+\frac{\varepsilon^{2}\left\|Q^{1 / 2}(A+B K) A^{-1} L C F\right\|^{2}}{\left(1-\varepsilon^{2}\right)\left(1-\|A-L C\|^{2}\right)} \phi_{W} .
\end{aligned}
$$

By summing (8), (26), (35), (41), (44), (55), and (58), obtain that

$$
J_{1}=\limsup _{T \rightarrow \infty} \frac{1}{T} \sum_{k=0}^{T-1} E\left[X^{\prime}(k) Q X(k)\right]
$$

$$
<\frac{1}{1-\|A-L C\|^{2}}
$$

$$
\times\left[\frac{1}{\left(1-\sqrt[n]{|A|^{2} / 4^{\bar{R}}}\right)\left(1-\|A+B K\|^{2}\right)}\right.
$$

$$
\begin{aligned}
& \times\left(\sqrt[n]{\frac{|A|^{2}}{4^{\bar{R}}}}\left\|Q^{1 / 2}(A+B K) A^{-1} L C F\right\|^{2}\right. \\
& +\frac{2\left\|F^{\prime}(L C)^{\prime}(A-L C)^{\prime} Q(A+B K) L C F\right\|}{\left(1-\|A+B K\|^{2}\right)\left(1-\left\|(A+B K)^{\prime}(A-L C)\right\|\right)} \\
& +\left\|Q^{1 / 2} F\right\|^{2} \\
& \left.+2 \sqrt[n]{\frac{|A|^{2}}{4^{\bar{R}}}}\left\|F^{\prime}(L C)^{\prime}(A+B K)^{\prime} Q A^{-1} L C F\right\|\right) \\
& +\left\|Q^{1 / 2} L C F\right\|^{2} \\
& \left.+\frac{2\left\|F^{\prime}(A-L C)^{\prime} Q L C F\right\|}{1-\left\|(A+B K)^{\prime}(A-L C)\right\|}\right] \phi_{W} .
\end{aligned}
$$

Now, further consider the case with $Q=I$, where $I$ denotes an identity matrix. Then, the equality above may reduce to

$$
\begin{aligned}
& \limsup _{T \rightarrow \infty} \frac{1}{T} \sum_{k=0}^{T-1} E\|X(k)\|^{2} \\
& <\frac{1}{1-\|A-L C\|^{2}} \\
& \times\left[\frac{1}{\left(1-\sqrt[n]{|A|^{2} / 4^{\bar{R}}}\right)\left(1-\|A+B K\|^{2}\right)}\right. \\
& \times\left(\sqrt[n]{\frac{|A|^{2}}{4^{\bar{R}}}\left\|(A+B K) A^{-1} L C F\right\|^{2}+\|L C F\|^{2}}\right. \\
& \left.+2 \sqrt[n]{\frac{|A|^{2}}{4^{\bar{R}}}}\left\|F^{\prime}(L C)^{\prime}(A+B K)^{\prime} A^{-1} L C F\right\|\right)
\end{aligned}
$$




$$
\begin{aligned}
& +\frac{2\left\|F^{\prime}(L C)^{\prime}(A-L C)^{\prime}(A+B K) L C F\right\|}{\left(1-\|A+B K\|^{2}\right)\left(1-\left\|(A+B K)^{\prime}(A-L C)\right\|\right)} \\
& \left.+\|F\|^{2}+\frac{2\left\|F^{\prime}(A-L C)^{\prime} L C F\right\|}{1-\left\|(A+B K)^{\prime}(A-L C)\right\|}\right] \phi_{W} .
\end{aligned}
$$

It means that the system (1) is stabilizable in the mean square sense (9) if the data rate $R$ satisfies the following condition:

$$
R>R_{\min }=\log _{2}|A|(\text { bits } / \text { sample }) .
$$

Remark 3. (i) It is shown in Theorem 2 that the system (1) is still stabilizable in the mean square sense (9) by employing a bit-allocation scheme on the basis of not the eigenvalues but the singular values of the system matrix. Furthermore, under the special condition, our result may reduce to the existing one in [1] and is not more conservative.

(ii) No assumption that there exists a real transformation matrix such that system matrix can be transformed to a diagonal matrix or a Jordan canonical form is made in this paper. Thus, the bit-allocation scheme presented here does work for more general systems.

Now, consider the classical LQG control problem for MIMO linear time-invariant systems with data-rate limitations and discuss the effect of the data rate on the achievable control performance. Namely, for a given data rate, the problem here is how to design the quantization, coding, and control scheme to achieve the optimal LQ cost. The performance objective to be considered here is the average infinite horizon quadratic cost:

$$
J_{2}=\limsup _{T \rightarrow \infty} \frac{1}{T} \sum_{k=0}^{T-1} E\left[X^{\prime}(k) Q X(k)+U^{\prime}(k) S U(k)\right],
$$

where $Q \in R^{n \times n}$ and $S \in R^{m \times m}$ are symmetric positive definite and $\left(A, Q^{1 / 2}\right)$ is observable.

Theorem 4. Consider the system (1). Assume that all eigenvalues of $A+B K$ and $A-L C$ lie inside the unit circle, and the magnitudes of all the singular values of system matrix $A$ are larger than 1 . The data rate of the channel satisfies the following inequality:

$$
R>R_{\min }=\log _{2}|A| \text { (bits/sample). }
$$

Then, the certainty equivalent control law of the form $U(t)=$ $K \widehat{X}_{o}(t)$ where

$$
K=-\left(B^{\prime} P B+S\right)^{-1} B^{\prime} P A
$$

and $P$ satisfies the Riccati equation

$$
P=A^{\prime}\left(P-P B\left(B^{\prime} P B+S\right)^{-1} B^{\prime} P\right) A+Q
$$

is optimal. The corresponding optimal LQ cost $J_{2}$ is given by

$$
\begin{aligned}
J_{2}= & \limsup _{T \rightarrow \infty} \frac{1}{T} \sum_{k=0}^{T-1} E\left[X^{\prime}(k) Q X(k)+U^{\prime}(k) S U(k)\right] \\
< & \frac{1}{1-\|A-L C\|^{2}} \\
& \times\left[\frac{\sqrt[n]{|A|^{2}}}{2^{2 R / n}-\sqrt[n]{|A|^{2}}}\right. \\
& \times\left(\left\|Q^{1 / 2} L C F\right\|^{2}+\left\|P^{1 / 2} A L C F\right\|^{2}+\left\|P^{1 / 2} L C F\right\|^{2}\right) \\
& \left.+\left\|P^{1 / 2} L C F\right\|^{2}+\left\|Q^{1 / 2} F\right\|^{2}\right] \phi_{W} .
\end{aligned}
$$

Proof. Notice that

$$
\widehat{X}_{o}(k+1)=X_{o}(k+1)-H^{\prime} V(k+1) .
$$

Substitute (6) into the equality above, and obtain that

$$
\begin{aligned}
\widehat{X}_{o}(k+1)= & A X_{o}(k)+B U(k) \\
& +L C D(k)-H^{\prime} V(k+1) \\
= & A \widehat{X}_{o}(k)+B U(k)+L C D(k) \\
& +A H^{\prime} V(k)-H^{\prime} V(k+1) .
\end{aligned}
$$

Furthermore, notice that

$$
X(k)=X_{o}(k)+D(k)=\widehat{X}_{o}(k)+H^{\prime} V(k)+D(k) .
$$

Then, Tatikonda et al. [7] addressed the classical LQG control problem under data-rate limitations and considered the same case. The optimal LQ cost decomposed into two terms: a full knowledge cost and a sequential rate distortion cost. However, the second term still depends on the steady state estimation error covariance. This paper further discusses the LQG control problem and presents a full knowledge cost. As stated in [7], the optimal steady state control law is a linear gain of the form $U(k)=K \widehat{X}_{o}(k)$ where

$$
K=-\left(B^{\prime} P B+S\right)^{-1} B^{\prime} P A
$$

and $P$ satisfies the Riccati equation:

$$
P=A^{\prime}\left(P-P B\left(B^{\prime} P B+S\right)^{-1} B^{\prime} P\right) A+Q .
$$


The optimal cost is given by

$$
\begin{aligned}
& J_{2}=\limsup _{T \rightarrow \infty} \frac{1}{T} \sum_{k=0}^{T-1} E\left[X^{\prime}(k) Q X(k)+U^{\prime}(k) S U(k)\right] \\
& =\limsup _{T \rightarrow \infty} \frac{1}{T} \sum_{k=0}^{T-1} E\left[\widehat{X}_{o}^{\prime}(t) Q \widehat{X}_{o}(t)+U^{\prime}(t) S U(t)\right] \\
& +\limsup _{T \rightarrow \infty} \frac{1}{T} \sum_{k=0}^{T-1} E\left[V^{\prime}(k) H Q H^{\prime} V(k)\right] \\
& +\limsup _{T \rightarrow \infty} \frac{1}{T} \sum_{k=0}^{T-1} E\left[D^{\prime}(k) Q D(k)\right] \\
& =\underset{T \rightarrow \infty}{\limsup } \frac{1}{T} \sum_{k=0}^{T-1} \operatorname{tr}\left[H A^{\prime} P A H^{\prime} \sum_{V(k)}\right] \\
& +\underset{T \rightarrow \infty}{\limsup } \frac{1}{T} \sum_{k=0}^{T-1} \operatorname{tr}\left[H P H^{\prime} \sum_{V(k+1)}\right] \\
& +\limsup _{T \rightarrow \infty} \frac{1}{T} \sum_{k=0}^{T-1} \operatorname{tr}\left[(L C)^{\prime} P L C \sum_{D(k)}\right] \\
& +\limsup _{T \rightarrow \infty} \frac{1}{T} \sum_{k=0}^{T-1} E\left[V^{\prime}(k) H Q H^{\prime} V(k)\right] \\
& +\limsup _{T \rightarrow \infty} \frac{1}{T} \sum_{k=0}^{T-1} E\left[D^{\prime}(k) Q D(k)\right] \\
& =\limsup _{T \rightarrow \infty} \frac{1}{T} \sum_{k=0}^{T-1} E\left[V^{\prime}(k) H A^{\prime} P A H^{\prime} V(k)\right] \\
& +\limsup _{T \rightarrow \infty} \frac{1}{T} \sum_{k=0}^{T-1} E\left[V^{\prime}(k+1) H P H^{\prime} V(k+1)\right] \\
& +\limsup _{T \rightarrow \infty} \frac{1}{T} \sum_{k=0}^{T-1} E\left[D^{\prime}(k)(L C)^{\prime} P L C D(k)\right] \\
& +\limsup _{T \rightarrow \infty} \frac{1}{T} \sum_{k=0}^{T-1} E\left[V^{\prime}(k) H Q H^{\prime} V(k)\right] \\
& +\limsup _{T \rightarrow \infty} \frac{1}{T} \sum_{k=0}^{T-1} E\left[D^{\prime}(k) Q D(k)\right] .
\end{aligned}
$$

Then, it needs to compute all the terms in the equation above. It follows from (31) that

$$
\varepsilon^{2}\left[H Q H^{\prime}\right]_{i, i} \sigma_{i}^{-2} E\left[z_{i}^{2}(k)\right]=\left[H Q H^{\prime}\right]_{i, i} E\left[v_{i}^{2}(k)\right] .
$$

Then, it holds that

$$
\begin{gathered}
\varepsilon^{2} E\left[Z^{\prime}(k) H\left(A^{-1}\right)^{\prime} Q A^{-1} H^{\prime} Z(k)\right] \\
=E\left[V^{\prime}(k) H Q H^{\prime} V(k)\right] .
\end{gathered}
$$

Furthermore, it follows from (30) that

$$
\begin{aligned}
& {\left[H Q H^{\prime}\right]_{i, i} \sigma_{i}^{-2} E\left[z_{i}^{2}(k+1)\right]} \\
& \quad=\left[H Q H^{\prime}\right]_{i, i} E\left[v_{i}^{2}(k)\right]+\left[(L C)^{\prime} Q L C\right]_{i, i} E\left[d_{i}^{2}(k)\right] .
\end{aligned}
$$

Then, it holds that

$$
\begin{aligned}
& E\left[Z^{\prime}(k+1) H\left(A^{-1}\right)^{\prime} Q A^{-1} H^{\prime} Z(k+1)\right] \\
& \quad=E\left[V^{\prime}(k) H Q H^{\prime} V(k)\right]+E\left\|Q^{1 / 2} L C D(k)\right\|^{2} .
\end{aligned}
$$

Substitute (74) into the equality above, and obtain that

$$
\begin{aligned}
E\left[Z^{\prime}(k+1) H\left(A^{-1}\right)^{\prime} Q A^{-1} H^{\prime} Z(k+1)\right] & \\
= & \varepsilon^{2} E\left[Z^{\prime}(k) H\left(A^{-1}\right)^{\prime} Q A^{-1} H^{\prime} Z(k)\right] \\
& +E\left\|Q^{1 / 2} L C D(k)\right\|^{2} .
\end{aligned}
$$

This implies that

$$
\begin{gathered}
\limsup _{T \rightarrow \infty} \frac{1}{T} \sum_{k=0}^{T-1} E\left[Z^{\prime}(k) H\left(A^{-1}\right)^{\prime} Q A^{-1} H^{\prime} Z(k)\right] \\
<\frac{1}{1-\varepsilon^{2}} E\left\|Q^{1 / 2} L C D(k)\right\|^{2} \\
<\frac{\left\|Q^{1 / 2} L C F\right\|^{2}}{\left(1-\varepsilon^{2}\right)\left(1-\|A-L C\|^{2}\right)} \phi_{W} .
\end{gathered}
$$

Thus, it holds that

$$
\begin{aligned}
& \limsup _{T \rightarrow \infty} \frac{1}{T} \sum_{k=0}^{T-1} E\left[V^{\prime}(k) H Q H^{\prime} V(k)\right] \\
&<\frac{\varepsilon^{2}\left\|Q^{1 / 2} L C F\right\|^{2}}{\left(1-\varepsilon^{2}\right)\left(1-\|A-L C\|^{2}\right)} \phi_{W} .
\end{aligned}
$$

Using the same techniques in the proof above, it can be shown that

$$
\begin{gathered}
\limsup _{T \rightarrow \infty} \frac{1}{T} \sum_{k=0}^{T-1} E\left[V^{\prime}(k) H A^{\prime} P A H^{\prime} V(k)\right] \\
<\frac{\varepsilon^{2}\left\|P^{1 / 2} A L C F\right\|^{2}}{\left(1-\varepsilon^{2}\right)\left(1-\|A-L C\|^{2}\right)} \phi_{W}, \\
\limsup _{T \rightarrow \infty} \frac{1}{T} \sum_{k=0}^{T-1} E\left[V^{\prime}(k+1) H P H^{\prime} V(k+1)\right] \\
<\frac{\varepsilon^{2}\left\|P^{1 / 2} L C F\right\|^{2}}{\left(1-\varepsilon^{2}\right)\left(1-\|A-L C\|^{2}\right)} \phi_{W} .
\end{gathered}
$$


Furthermore, it follows from (7) that

$$
\begin{aligned}
& \limsup _{T \rightarrow \infty} \frac{1}{T} \sum_{k=0}^{T-1} E\left[D^{\prime}(k+1)(L C)^{\prime} P L C D(k+1)\right] \\
& <\|A-L C\|^{2} \limsup _{T \rightarrow \infty} \frac{1}{T} \sum_{k=0}^{T-1} E\left[D^{\prime}(k)(L C)^{\prime} P L C D(k)\right] \\
& +\left\|P^{1 / 2} L C F\right\|^{2} \phi_{W} .
\end{aligned}
$$

Then, it holds that

$$
\begin{gathered}
\limsup _{T \rightarrow \infty} \frac{1}{T} \sum_{k=0}^{T-1} E\left[D^{\prime}(k)(L C)^{\prime} P L C D(k)\right] \\
<\frac{\left\|P^{1 / 2} L C F\right\|^{2}}{1-\|A-L C\|^{2}} \phi_{W} \cdot
\end{gathered}
$$

Substitute (8), (41), (79), (80), and (82), and obtain that

$$
\begin{aligned}
J_{2}= & \limsup _{T \rightarrow \infty} \frac{1}{T} \sum_{k=0}^{T-1} E\left[X^{\prime}(k) Q X(k)+U^{\prime}(k) S U(k)\right] \\
< & \frac{1}{1-\|A-L C\|^{2}} \\
& \times\left[\frac{\sqrt[n]{|A|^{2}}}{2^{2 R / n}-\sqrt[n]{|A|^{2}}}\right. \\
& \times\left(\left\|Q^{1 / 2} L C F\right\|^{2}+\left\|P^{1 / 2} A L C F\right\|^{2}+\left\|P^{1 / 2} L C F\right\|^{2}\right) \\
& \left.+\left\|P^{1 / 2} L C F\right\|^{2}+\left\|Q^{1 / 2} F\right\|^{2}\right] \phi_{W} .
\end{aligned}
$$

Remark 5. (i) This section presents an explicit expression on the tradeoff between the LQ cost and the data rate. Namely, for the given data rate, it is possible to compute the achievable performance, and for the given LQ cost, it is also possible to compute the minimum data rate needed to achieve the control performance.

(ii) It also states in Theorem 4 that the larger data rate may lead to the better control performance. However, the control performance cannot become better when the data rate is large enough. For the case with $R \rightarrow \infty$, the corresponding optimal LQ cost $J_{2}$ is given by

$$
\lim _{R \rightarrow \infty} J_{2}<\frac{1}{1-\|A-L C\|^{2}}\left(\left\|P^{1 / 2} L C F\right\|^{2}+\left\|Q^{1 / 2} F\right\|^{2}\right) \phi_{W} .
$$

\section{Numerical Example}

This paper investigates a class of networked control problems which arises in the coordinated motion control of

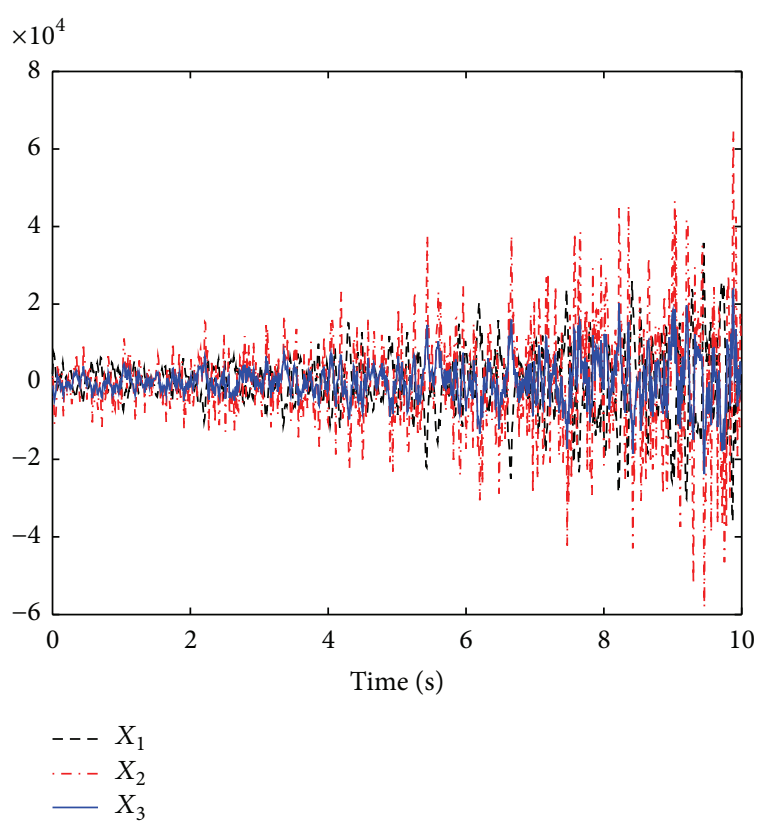

FIGURE 2: First, let the data rate $R=200$ (bits/s), which is smaller than the lower bound on the data rate given by Theorem 4 . A quantization, coding, control scheme on the basis of such data rate is implemented. The corresponding simulation is given in Figure 2. It is shown that the system is unstable. The system state responses with $R=200$ (bits/s).

autonomous and semiautonomous mobile agents, for example, unmanned air vehicles (UAVs), unmanned ground vehicles (UGVs), and unmanned underwater vehicles (UUVs), and addresses the LQG control under data-rate limitations. To illustrate the effectiveness of the schemes presented in this paper, this section presents a practical example, where three of the states of an unmanned air vehicle evolve in discretetime according to

$$
\begin{aligned}
X(k+1)= & {\left[\begin{array}{lll}
2.3153 & 2.4614 & 0.2125 \\
0.3421 & 3.3312 & 0.3443 \\
0.7328 & 0.2127 & 2.4575
\end{array}\right] X(k) } \\
& +\left[\begin{array}{ll}
1.1342 & 2.3412 \\
1.1545 & 2.7612 \\
0.2344 & 7.1251
\end{array}\right] U(k) \\
& +\left[\begin{array}{ll}
5.6542 \\
1.3432 \\
0.7823
\end{array}\right] W(k), \\
Y(k)= & {\left[\begin{array}{lll}
3.7834 & 1.8392 & 2.8934 \\
1.6723 & 1.8924 & 3.7824
\end{array}\right] X(k) . }
\end{aligned}
$$

Here, let the initial position $X(0)=\left[\begin{array}{lll}5000 & -5000 & 1000\end{array}\right]^{\prime}$ and $\phi_{W}=50$. The control performance will be examined in four cases.

Secondly, let the data rate $R=400$ (bits/s), which is larger than the lower bound on the data rate given by Theorem 4 . A quantization, coding, control scheme on the basis of such data rate is implemented to stabilize the unstable plant. 


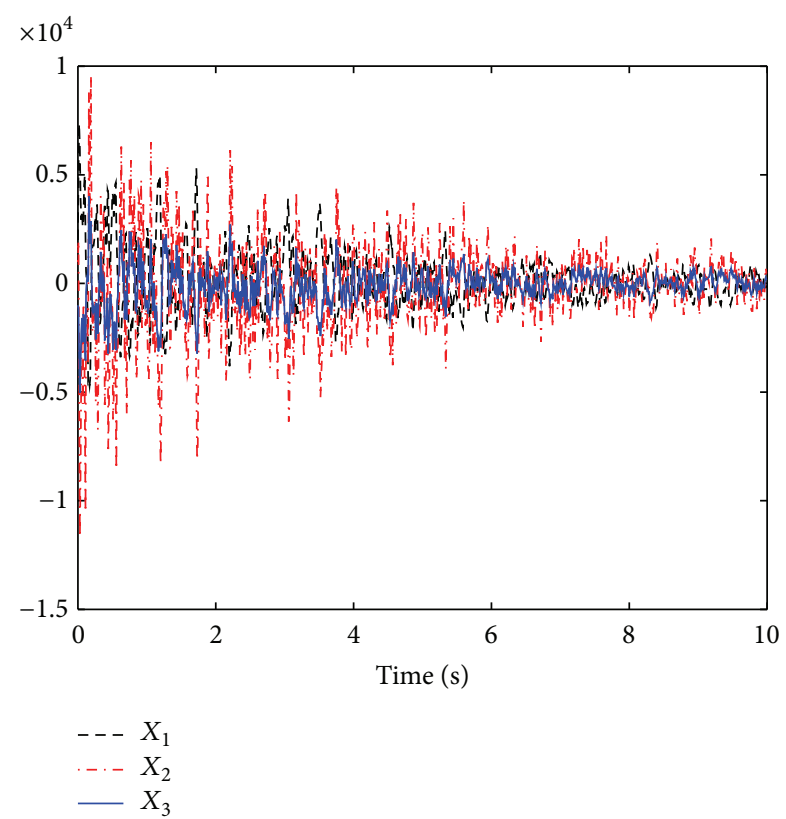

FIgURE 3: The system state responses with $R=400$ (bits/s).

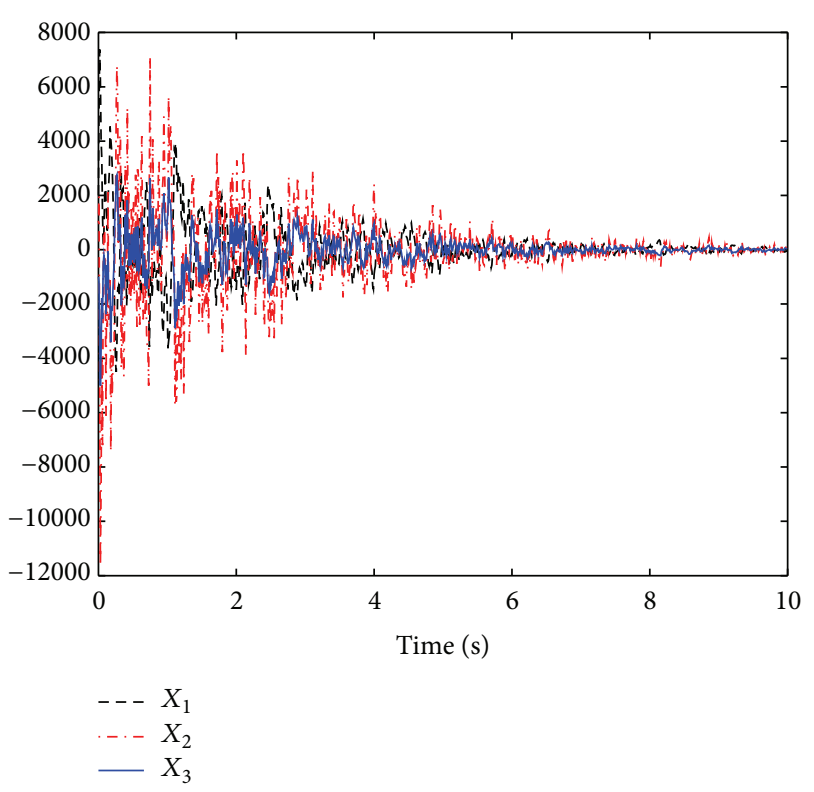

FIGURE 4: The system state responses with $R=800$ (bits/s).

The simulation is given in Figure 3. It is shown that the system is stabilizable, and the optimal LQ cost is given by $J_{2}=$ 2386.23. However, it is impossible to achieve the given control objective when the data rate is too small.

Then, the data rate is enlarged in order to achieve the given control objective. Let the data rate $R=800$ (bits/s). The corresponding simulation is given in Figure 4. The optimal LQ cost is given by $J_{2}=352.51$. It states that the larger data rate leads to better control performance.

Finally, let the data rate $R=1600$ (bits/s), which is far larger than the lower bound on the data rate given by Theorem 4. The quantization, coding, control scheme on

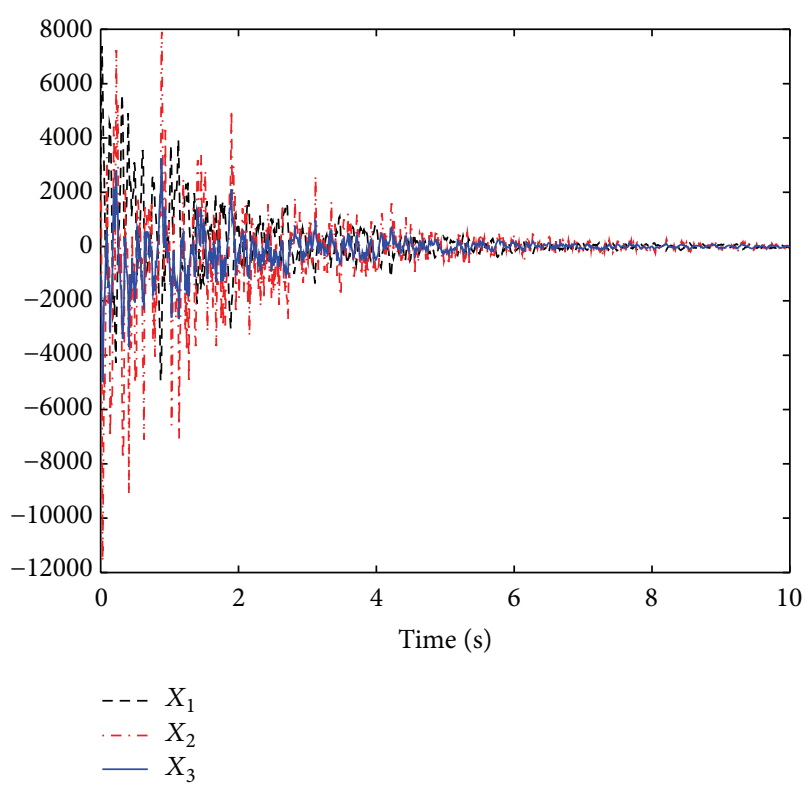

FIGURE 5: The system state responses with $R=1600$ (bits/s).

the basis of such data rate is implemented to stabilize the unstable plant. The corresponding simulation is given in Figure 5. The optimal LQ cost is given by $J_{2}=351.76$. It states that it is difficult to improve the control performance by further enlarging the data rate.

\section{Conclusion}

This paper addressed the LQG control problem for MIMO discrete-time linear systems over digital communication channels with limited data rates. The data-rate limitations often lead to the poor control performance. A key issue is how to design the quantization, coding, and control scheme to achieve the minimum data rate for stabilization and some control performances. A bit-allocation scheme on the basis of the singular values of the system matrix was presented. It was shown in our results that there exists the inherent tradeoff between the LQ cost and the data rate. The simulation results have illustrated such tradeoff. The study of LQG control for nonlinear system under data-rate limitations will be our future work.

\section{Conflict of Interests}

The authors declare that there is no conflict of interests regarding the publication of this paper.

\section{Acknowledgments}

This work is partially supported by China Postdoctoral Science Foundation funded project (no. 2013M530134) and the Open Foundation of Automatic Weapons and Ammunition Engineering Key Disciplines of Shenyang Ligong University (no. 4771004kfx02). The authors also gratefully acknowledge the helpful comments and suggestions of the reviewers, which have improved the presentation. 


\section{References}

[1] J. Baillieul and P. Antsaklis, "Control and communication challenges in networked real-time systems," Proceedings of the IEEE, vol. 95, no. 1, pp. 9-28, 2007.

[2] G. N. Nair, F. Fagnani, S. Zampieri, and R. J. Evans, "Feedback control under data rate constraints: an overview," Proceedings of the IEEE, vol. 95, no. 1, pp. 108-137, 2007.

[3] J. Baillieul, "Feedback designs in information based control," in Stochastic Theory and Control Proceedings of a Workshop Held in Lawrence, Kansas, B. Pasik-Duncan, Ed., pp. 35-57, Springer, New York, NY, USA, 2001.

[4] G. N. Nair and R. J. Evans, "Stabilizability of stochastic linear systems with finite feedback data rates," SIAM Journal on Control and Optimization, vol. 43, no. 2, pp. 413-436, 2004.

[5] R. W. Brockett and D. Liberzon, "Quantized feedback stabilization of linear systems," IEEE Transactions on Automatic Control, vol. 45, no. 7, pp. 1279-1289, 2000.

[6] V. Borkar and S. K. Mitter, "LQG control with communication constraints," in Communications, Computation, Control, and Signal Processing: A Tribute to Thomas Kailath, pp. 1255-1261, Kluwer Academic Publishers, Norwell, Mass, USA, 1997.

[7] S. Tatikonda, A. Sahai, and S. Mitter, "Stochastic linear control over a communication channel," IEEE Transactions on Automatic Control, vol. 49, no. 9, pp. 1549-1561, 2004.

[8] O. C. Imer and T. Başar, "Optimal control with limited controls," in Proceedings of the American Control Conference, pp. 298-303, June 2006.

[9] L. Schenato, B. Sinopoli, M. Franceschetti, K. Poolla, and S. S. Sastry, "Foundations of control and estimation over lossy networks," Proceedings of the IEEE, vol. 95, no. 1, pp. 163-187, 2007.

[10] P. Bommannavar and T. Başar, "Optimal control with limited control actions and lossy transmissions," in Proceedings of the 47th IEEE Conference on Decision and Control (CDC '08), pp. 2032-2037, Cancún, Mexico, December 2008.

[11] C. D. Charalambous and A. Farhadi, "LQG optimality and separation principle for general discrete time partially observed stochastic systems over finite capacity communication channels," Automatica, vol. 44, no. 12, pp. 3181-3188, 2008.

[12] D. Georges, G. Besancon, and J.-F. Dulhoste, "A decentralized optimal LQ state observer based on an augmented Lagrangian approach," Automatica, vol. 50, no. 5, pp. 1451-1458, 2014.

[13] Y.-L. Wang and Q.-L. Han, "Modelling and controller design for discrete-time networked control systems with limited channels and data drift," Information Sciences, vol. 269, pp. 332-348, 2014.

[14] T. Cover and J. Thomas, Elements of Information Theory, Wiley, New York, NY, USA, 2006. 


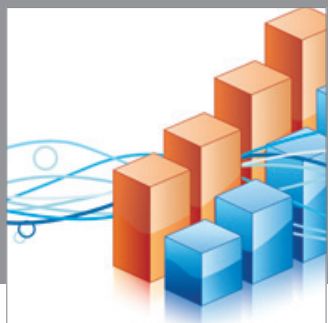

Advances in

Operations Research

mansans

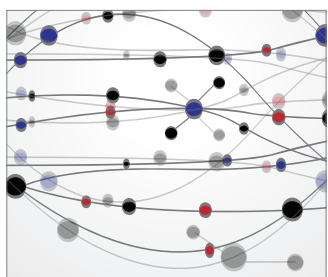

The Scientific World Journal
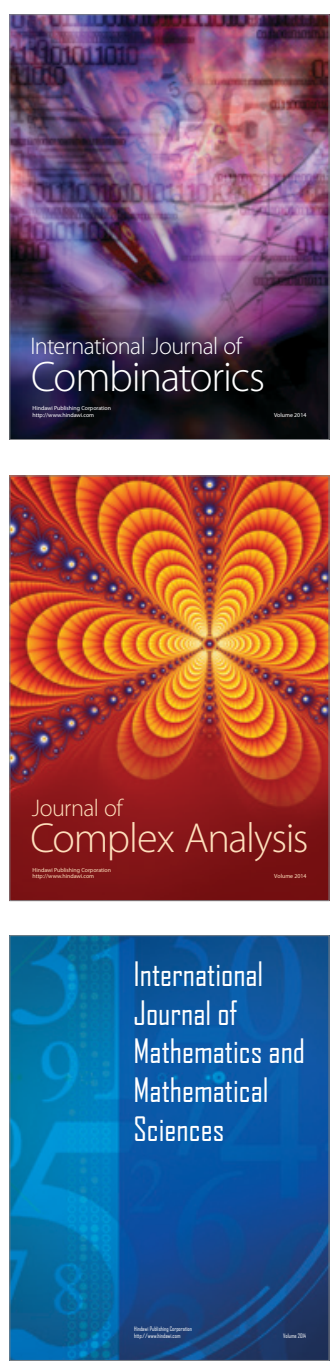
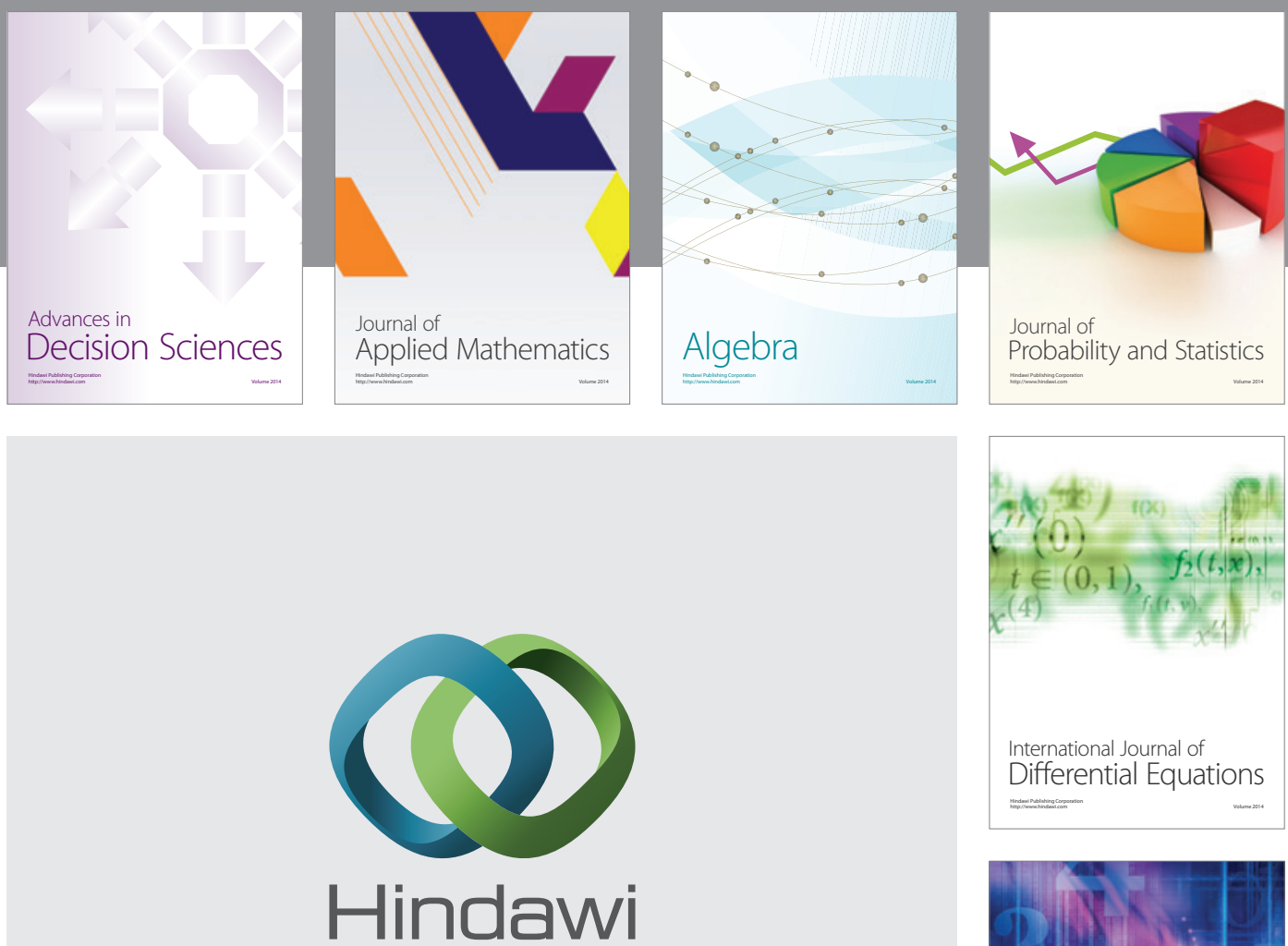

Submit your manuscripts at http://www.hindawi.com
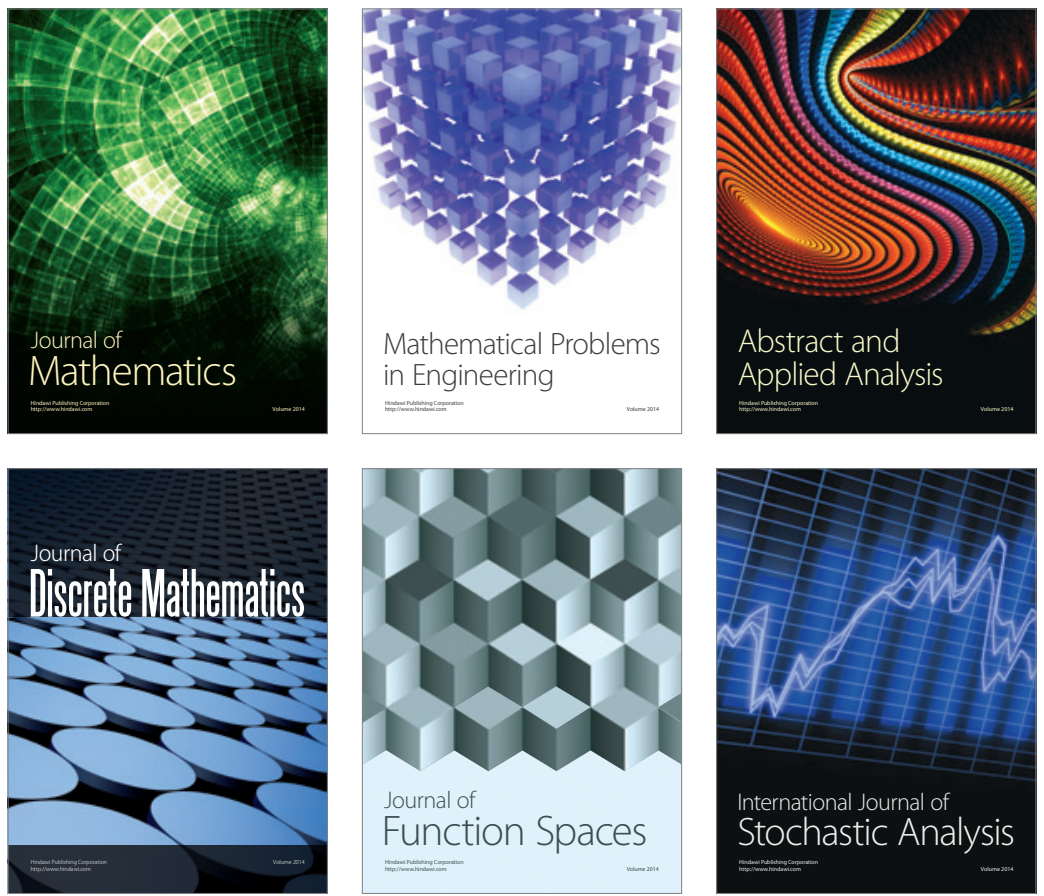

Journal of

Function Spaces

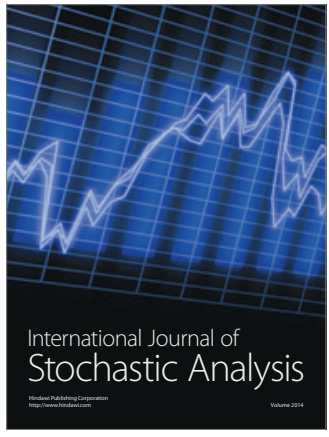

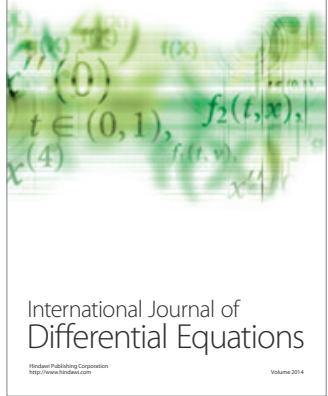
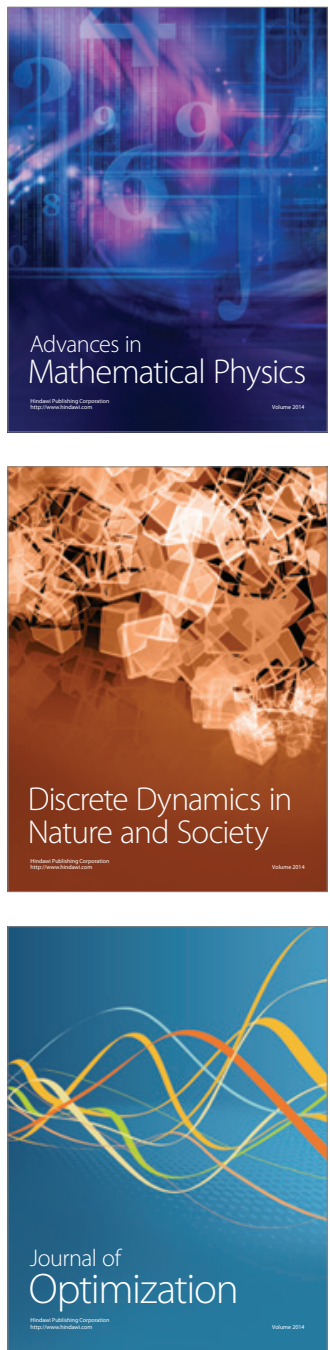